OPEN ACCESS

Edited by:

Stephanie Jost,

Duke University, United States

Reviewed by:

Seth Guller,

Yale University, United States

Leticia Reyes,

University of Wisconsin-Madison,

United States

*Correspondence:

Megan C. Sharps

megan.sharps@manchester.ac.uk

Specialty section: This article was submitted to

Translational Virology,

a section of the journal

Frontiers in Virology

Received: 11 November 2021

Accepted: 06 January 2022

Published: 08 February 2022

Citation:

Sharps MC, Garrod A, Aneni E, Jones CJP, Batra G and Heazell AEP

(2022) Placental Macrophages

Following Maternal SARS-CoV-2 Infection in Relation to Placental

Pathology. Front. Virol. 2:813312.

doi: 10.3389/fviro.2022.813312

\section{Placental Macrophages Following Maternal SARS-CoV-2 Infection in Relation to Placental Pathology}

\author{
Megan C. Sharps ${ }^{1 *}$, Ainslie Garrod ${ }^{1}$, Emmanuel Aneni ${ }^{1}$, Carolyn J. P. Jones ${ }^{1}$, Gauri Batra ${ }^{2}$ \\ and Alexander E. P. Heazell ${ }^{1,3}$ \\ ${ }^{1}$ Tommy's Maternal and Fetal Health Research Centre, Faculty of Biology, Medicine and Health, St Mary's Hospital, \\ University of Manchester, Manchester, United Kingdom, ${ }^{2}$ The Department of Paediatric and Perinatal Pathology, Royal \\ Manchester Children's Hospital, Manchester University Hospitals NHS Foundation Trust, Manchester, United Kingdom, \\ ${ }^{3}$ Manchester Academic Health Science Centre, St. Mary's Hospital, Manchester University NHS Foundation Trust, \\ Manchester, United Kingdom
}

Introduction: In December 2019, a novel coronavirus, SARS-CoV-2, was identified. Whilst pregnant women appear to be at risk of severe infection, pre-term birth, and stillbirth, it is unclear whether placental dysfunction is a consistent feature of maternal SARS-CoV-2 infection during pregnancy. We aim to describe the immune response in placentas of women who had COVID-19 infection during pregnancy and investigate whether there are any associated morphological changes.

Methods: The placentas of women testing positive for COVID-19 during their pregnancy were compared to contemporaneous controls who were not known to have had COVID-19 during pregnancy. Samples of each placenta were sent for histopathological analysis or underwent immunohistochemical staining for CD163, CD20, CD3, CD31, and SARS-CoV-2 spike protein. A subset of samples were sent for transmission electron microscopy.

Results: There was a significant increase in the number of $\mathrm{CD}_{163^{+}}$macrophages in the Post COVID group $(p=0.0020)$. There was no difference in the percentage of $\mathrm{CD}^{+}$, $\mathrm{CD}_{20}{ }^{+}$cells, but there was an increase in placental vascularity in the Post COVID group compared to controls $(p=0.026)$.

There were no structural differences observed between the samples sent for EM analysis. However, one of the placentas from the Post COVID group was seen to have several large sub-apical vacuoles in the syncytiotrophoblast. We did not observe any virions within the vacuoles and SARS-CoV-2 spike protein staining was negative for the sample. Histopathological investigations indicated that there was no specific placental pathology caused by maternal COVID-19 infection in this cohort of samples.

Conclusions: This study did not confirm previous studies which describe a possible increase in cases of both maternal and fetal vascular malperfusion, and placentitis in women who had COVID-19, which were seen in association with adverse pregnancy outcomes. It remains unclear whether observed abnormalities are caused by maternal infection, or whether maternal infection exacerbates existing placental pathology; understanding why some placentas generate these abnormalities is a key goal.

Keywords: COVID-19, SARS-CoV-2, placenta, histopathology, macrophages 


\section{INTRODUCTION}

SARS-CoV-2 was first detected in Wuhan, China, in December 2019. The resulting disease COVID-19, became a worldwide pandemic, with nearly 200 million people catching the virus which to date has claimed the lives of over four million people (1). A systematic review of international studies suggests that SARS$\mathrm{CoV}-2$ infection during pregnancy is associated with an increased risk of maternal and fetal deaths (2); data regarding spontaneous pre-term births are equivocal.

Although currently there appears to be no specific placental pathology associated with maternal SARS-CoV-2 infection (3), several cases of placentitis have been described in association with stillbirth, suggesting that placental inflammation may occur after SARS-CoV-2 infection and could be associated with fetal death. There is a paucity of research investigating the presence of macrophages, and other immune cells in the placenta following infection. Whilst macrophages form part of the innate immune system, $\mathrm{T}$ and $\mathrm{B}$ lymphocytes form the adaptive immune system which can tailor the immune response to the pathogen (4). Placental macrophages, otherwise known as Hofbauer cells, reside in the stromal core of the placental villi (5). In other infectious diseases, such as ZIKA virus, placental macrophages are thought to be a reservoir for viral replication (6).

There are conflicting data available as to whether the number of Hofbauer cells are increased following maternal SARS-CoV2 infection. Whilst some studies have found no increase in Hofbauer cell number $(7,8)$, other studies report an increase (9). There are limited data regarding the effect of SARS-CoV-2 infection on $\mathrm{T}$ or $\mathrm{B}$ lymphocyte numbers, although one study reported no difference following maternal infection (8).

As there are still no conclusive data as to whether maternal SARS-CoV-2 infections leads to placental pathology, we aimed to investigate if there was an increase in the number of immune cells (macrophages, $\mathrm{T}$ and $\mathrm{B}$ lymphocytes) and an altered placental morphology in the placentas of women who had tested positive for SARS-CoV-2 during their pregnancy, or those who tested positive shortly before or at delivery, compared to healthy controls. We also performed histopathological analyses on the placentas, to identify if any pathologies were more likely to be present following maternal SARS-CoV-2 infection compared to controls.

\section{MATERIALS AND METHODS}

Placentas were collected from women who tested positive for COVID-19 $<12$ days prior to delivery or at delivery (Active COVID-19) and women who had tested positive $>12$ days prior to delivery (Post COVID-19). Women who to their knowledge had never had COVID-19 infection during their pregnancy and who had a negative PCR test on admission to hospital for delivery as per hospital policy were included in the control group. Women in the Active and Post COVID-19 groups had PCR tests following symptom onset, or had a positive antibody test. None of the women included in the study had severe symptoms requiring admission to hospital or enhanced care. Due to the low availability of samples, participants were included in spite of any maternal pregnancy complications or if the fetus was small or large for gestational age. Written, informed consent was given by all participants (18/NW/0451).

\subsection{Immunohistochemistry}

Sections of formalin fixed, paraffin-embedded placenta and umbilical cord were immunostained with the following antibodies: CD163 for macrophages (BioRad, $1 \mathrm{mg} / \mathrm{mL}$ at 1:100), CD3 for T lymphocytes (Dako, $138 \mathrm{mg} / \mathrm{L}$ at 1:100) CD20 for B lymphocytes (Invitrogen, concentration not determined at 1:200) or CD31 for endothelial cells (Dako, $201 \mathrm{mg} / \mathrm{mL}$ at 1:50). Tissue sections were also stained with haematoxylin and eosin ( $\mathrm{H} \& \mathrm{E})$ to allow for structural analysis. Immunostaining for the SARS-CoV-2 spike protein (Stratech (1:1000)) was performed at the Department of Pathology, Alder Hay Hospital, Liverpool on a Dako automatic platform following their standard histopathology staining procedure. A placenta previously identified as testing positive for SARS-CoV-2 was used as a positive control sample for every staining run.

Slides were imaged using Pannoramic 250 slide scanner (3D HISTECH, Bioimaging Facility, University of Manchester). Positive staining was analyzed using QuPath (version 0.2.3) as previously described (10). Briefly, CD163 analysis was performed using the positive cell detection function in QuPath, areas of quantifiable tissue were highlighted and thresholds were set to detect positive $\mathrm{DAB}$ staining compared to haematoxylin staining. Identification of $\mathrm{T}$ and $\mathrm{B}$ lymphocytes was performed by selection of 10 random $1 \times 1 \mathrm{~mm}$ regions of tissue, manually counting the number of lymphocytes and automatic detection of haematoxylin-stained nuclei. Vascularity was determined by calculating the area of $\mathrm{CD} 31$ positive $\mathrm{DAB}$ staining as a percentage of tissue area. Placental macrophages $\left(\mathrm{CD}_{163^{+}}\right)$, $\mathrm{T}\left(\mathrm{CD}^{+}\right)$, and $\mathrm{B}\left(\mathrm{CD} 20^{+}\right)$lymphocytes were expressed as a percentage of number of nuclei. Quantification of staining was performed excluding any areas of folded or poor quality tissue and a minimum of two tissue sections per tissue per immunostain were analyzed.

Histopathological investigations were performed by a consultant perinatal pathologist (GB) who was blinded to the maternal COVID-19 status. Histopathological description and diagnosis of the placentas was made following the Amsterdam Placental Workshop Group Consensus Statement (11).

\subsection{Transmission Electron Microscopy}

Approximately $2 \mathrm{~mm}^{2}$ sized pieces of placental villous tissue were fixed in $4 \%$ paraformaldehyde (PFA) and $2.5 \%$ gluteraldehyde in 0.1 M HEPES (4-(2-hydroxyethyl)-1-piperazineethanesulfonic acid) for $24 \mathrm{~h}$. The samples were then transferred to $0.5 \%$ PFA until they were processed by the staff in the EM Core Facility in the Faculty of Biology, Medicine and Health, University of Manchester. Semi-thin sections were produced by CJPJ.

Two samples from each group were chosen for transmission electron microscopy (TEM), due to their percentage of CD163 ${ }^{+}$ cells being close to the median for their group. Samples were analyzed on an FEI Tecnai12 BioTwin transmission electron microscope (TEM). 


\subsection{Statistics}

Statistical analyses were performed in Graphpad Prism (Version 8, Jolla, CA, USA). Mann-Whitney U or Kruskal-Wallis, with Dunn's post hoc, or Fischer's exact tests were performed as appropriate. The threshold of statistical significance was set at $p<0.05$.

\section{RESULTS}

\subsection{Participant Demographics}

\subsubsection{Maternal Data}

The demographic characteristics of the women included in the study were similar between the groups (Table 1). The parity of the women in the control group was significantly higher than that of the women in the Active and Post COVID groups ( $p=$ 0.03 and 0.007 , respectively) than controls. In the control group, approximately two thirds of women were Caucasian, compared to $55 \%$ in the Post COVID group and $38 \%$ in the Active COVID group.

Due to the poor availability of samples and comparatively low rates of COVID-19 infection in pregnant women (who were advised to shield in the lockdown periods), women were included in the study regardless of any co-morbidities. In the Active COVID group, one woman had pre-existing diabetes and chronic hypertension, two women were Rhesus negative, one having additional gestational diabetes. In the Post COVID group, one woman had hyperthyroidism and a second woman had recurrent reduced fetal movements (RFM). One woman in the control group also had recurrent RFM.

The mean gestation at maternal infection was 271 and 202 days for Active COVID and Post COVID pregnancies $(p>0.99$ and $p<0.0001$, respectively, compared to controls), although the exact date of a positive COVID test was only known for 18 Post COVID women. The median interval between a positive COVID-19 test result and delivery was 2.5 days (range $0-12$ days) in the Active COVID group $(p=0.0011)$ and 76 days (range $19-211$ days) in the Post COVID group $(p<0.0001)$.

There were some differences in the method of delivery with $50 \%$ of women being delivered by Cesarean section in the Active COVID group, compared to 55\% in the Post COVID group and $81 \%$ of the control group.

When the notes of one participant in the control group were reviewed to obtain missing data, a positive COVID-19 test the day after delivery was found, with an inconclusive test on the

TABLE 1 | Participant demographics for Active COVID, Post COVID, and control groups.

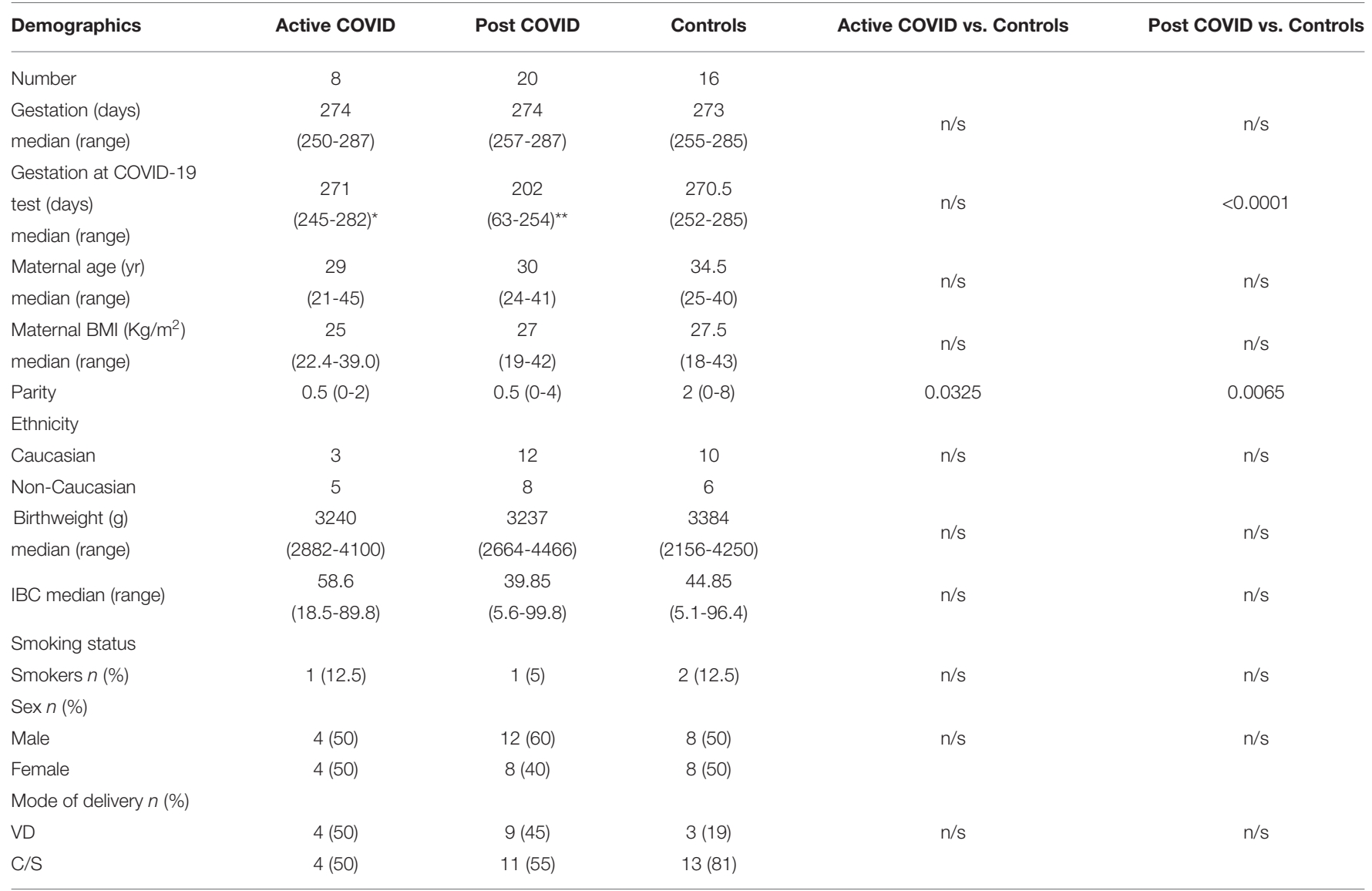

Statistics: Kruskal-Wallis or Fisher's exact test. Significance level $p<0.05 . I B C=$ individualized birthweight centile, VD = vaginal delivery, $C / S=C e s a r e a n$ section. ${ }^{*}$ one participant tested positive for COVID-19 one day post delivery, value recorded as 0 for purposes of analyses. ${ }^{\star *} t w o$ women self-reported a positive test result, exact date of test is unknown. 
day of delivery. Therefore, this participant was moved to the Active COVID group, and the positive test was recorded as date of delivery for the purposes of analyses.

\subsubsection{Neonatal Data}

A summary of neonatal data can be found in Table 1. All infants were live-born, with $50 \%$ of infants being male in the Active COVID group, compared to $60 \%$ in the Post COVID and 50\% in the healthy control groups. The median gestational age at delivery of infants from the Active COVID group was 274 days, whilst in the Post COVID and normal pregnancies, median gestational age were 274 and 273 days, respectively. There was no significant difference in the median birth weight of the infants between groups (Active COVID 3240g, Post COVID 3237g, control group 3384g).

No infants were born $\leq 10^{\text {th }}$ or $\geq 90^{\text {th }}$ individualized birthweight centile (IBC) in the Active COVID group. In contrast, four infants were born $\leq 10^{\text {th }}$ IBC in the Post COVID group, and five were born $\geq 90^{\text {th }}$, whilst two infants were born $\geq 90^{\text {th }}$ IBC and two $\leq 10^{\text {th }}$ IBC in the healthy control group. No infants in any group were born $\leq 5^{\text {th }} \mathrm{IBC}$, and, therefore, classed as having fetal growth restriction (FGR).

\subsection{Histopathology Reports}

Histopathology reports were obtained for 41 placentas. A summary of results can be found in Table 2. Full results can be found in Supplementary Table 1.

The most common observations were excessive syncytial knots, distal villous hypoplasia and villous immaturity. Villous dysmaturity was identified in one of the Active COVID placentas, three Post COVID placentas, and two of the negative control group placentas. One placenta from the Post COVID group had inflammation and one had infarcts. One of the negative controls had sub-chorionic chronic histiocytic inter-villositis and one Active COVID placenta had a chorangiosis-like appearance. There were no significant differences in the presence of histopathological lesions in placentas from the Active of Post COVID groups compared to healthy negative controls.

TABLE 2 | Summary of histopathology findings in placentas from Active COVID, Post COVID, and healthy control groups.

\begin{tabular}{lcccc}
\hline Pathology & $\begin{array}{c}\text { Active } \\
\text { Covid }\end{array}$ & $\begin{array}{c}\text { Post } \\
\text { covID }\end{array}$ & $\begin{array}{c}\text { Healthy } \\
\text { control }\end{array}$ & $\boldsymbol{p}$ value \\
\hline Excessive syncytial knots n (\%) & $5(71)$ & $10(56)$ & $8(50)$ & 0.63 \\
Distal villous hypoplasia n (\%) & $4(57)$ & $8(44)$ & $3(19)$ & 0.14 \\
Villous immaturity n (\%) & $2(29)$ & $6(33)$ & $6(38)$ & 0.91 \\
Villous dysmaturity n (\%) & $1(14)$ & $3(17)$ & $2(13)$ & 0.94 \\
Chorangiosis-like appearance n (\%) & $1(14)$ & 0 & 0 & 0.083 \\
Sub-chorionic chronic & 0 & 0 & $1(6)$ & 0.45 \\
histiocytic intervillositis n(\%) & & & & \\
Calcifications n (\%) & 0 & $2(11)$ & 0 & 0.26 \\
Inflammation n (\%) & 0 & $1(6)$ & 0 & 0.52 \\
\hline
\end{tabular}

Summary of histopathological lesions identified in placentas from the Active COVID $(n=$ 7), Post COVID $(n=18)$, and healthy controls $(n=16)$. Two Post COVID placentas were not available for analysis. Chi-squared test, $p<0.05$ considered statistically significant.

\subsection{Placental Histochemical Analysis}

\subsubsection{Immune Cells}

Analysis of the percentage of intra-villous $\mathrm{CD}_{163}{ }^{+}$Hofbauer cells found that there was a significant increase in the placentas from mothers who had tested positive for SARS-CoV-2 during their pregnancy (Post COVID) compared to controls $(p=0.002)$ (Figure 1A). There was no difference between the fetal sex within each group (data not shown). Representative images can be seen in Figure 1D.

Analysis of the percentage of both $\mathrm{CD}^{+}$and $\mathrm{CD} 20^{+}$ lymphocytes found that there was no difference between the placentas of women who had COVID-19 and those who had not (Figures 1B,C). There was no difference when the groups were split by fetal sex (data not shown). Representative images can be seen in Figures 1E,F.

\subsubsection{Vascularity}

There was a statistically significant increase in the percentage area of $\mathrm{CD}_{3} 1^{+}$endothelial cells in the placentas of the Post COVID group compared to controls $(\mathrm{p}=0.026)$. There was no difference observed in the Active COVID group compared to the control group $(\mathrm{p}=0.06)$ (Figure 2$)$.

\subsubsection{SARS-CoV-2 Spike Protein Staining}

Forty one placentas were sent for SARS-CoV-2 spike protein staining. None of the placentas stained positive for the spike protein; there was one area of staining in the syncytiotrophoblast of a placenta from the Active COVID group, but we could not be confident that this was not artefactual as there was staining of the stroma (Figures $\mathbf{3 A}, \mathbf{B}$, positive and negative controls Figures 3C,D).

\subsection{Ultrastructural Analysis}

A subset of samples were sent for ultrastructural analysis by TEM. There were no specific morphological abnormalities observed in either of the COVID-19 groups compared to controls.

In the Active COVID group, one sample had numerous dilated cisternae of the endoplasmic reticulum (Figure 4A). However, the rest of the samples looked healthy with normal basal lamina and mitochondria apart from one sample with an area of degenerate syncytium (Figure 4B) lacking normal microvilli. Another Active COVID sample had widely dilated cisternae of rough endoplasmic reticulum and some apical vacuoles with cytoplasmic contents; mitochondria were swollen and there were several cytoplasmic lamellar bodies. The basal lamina was thickened and the underlying Hofbauer cell had multiple inclusions. The capillary looked normal although the basal lamina was multi-layered, which has previous been observed in diabetes and Rhesus incompatibility and is a considered to be a sign of high cell turnover (12) (Figure 4C). Multiple ferritin particles were observed in Hofbauer cell inclusions (Figure 4D).

One placenta from the Post COVID group had several large sub-apical vacuoles in the syncytiotrophoblast (Figure 4E). The same sample had fibrotic stroma, a thick basal lamina and some malformed, swollen microvilli with another area of microvillous breakdown (Figure 4F). Another sample contained 


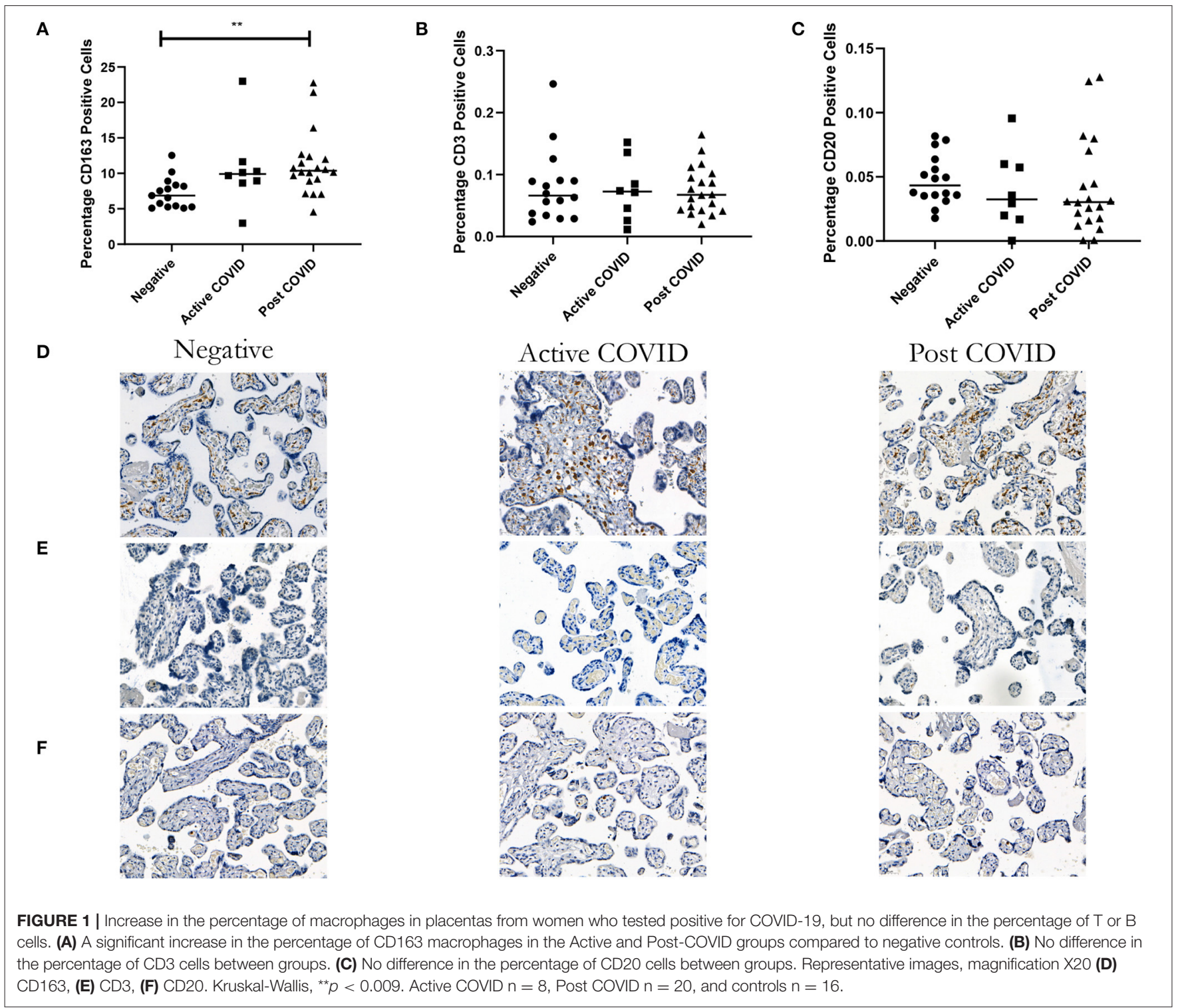

small capillaries for gestational age (Figure 4G) and alongside a thickened basal lamina, the tips of the microvilli were swollen (Figure 4H).

Almost all the samples had lamellar bodies (noted in Figures $4 \mathbf{H}, \mathbf{I}$ by ${ }^{*}$ ), but this may be an artifact of fixation. Other observations included small capillaries in a term terminal villus in a healthy control, whilst another control had numerous infoldings/interdigitation in the syncytium basal plasma membrane and the basal lamina was thickened and cribriform (Figure 4I). The former has been observed in many pathological conditions including pre-eclampsia and maternal hypertension while basal lamina thickening is a very non-specific response and has been described in most pathologies (13). Additionally, in controls, focal sites of necrosis with a total loss of microvilli and breakdown of the apical membrane and washed out cytoplasm were occasionally found (Figure 4J).

\section{DISCUSSION}

Whether placental pathology is associated or not with maternal SARS-COV-2 infection is of interest to obstetricians, histopathologists, and scientists attempting to delineate the effects of SARS-CoV-2 infection on pregnancy outcome. Our study has shown a significant increase in the percentage of $\mathrm{CD}_{163}{ }^{+}$macrophages in the Post COVID group compared to controls. We have previously shown an increase in intra-villous macrophages in several placental complications, including villitis of unknown etiology (VUE) (14) in the placentas of infants with a decreased growth rate, but not in those classified as FGR, which were thought to be small-for-gestational-age (SGA) (15). However, in contrast to previous studies, the observed increase in $\mathrm{CD}_{163^{+}}$cells in this current study is not associated with low birth weight, with no infants being classed FGR ( $\leq 3^{\text {rd }}$ IBC) and 


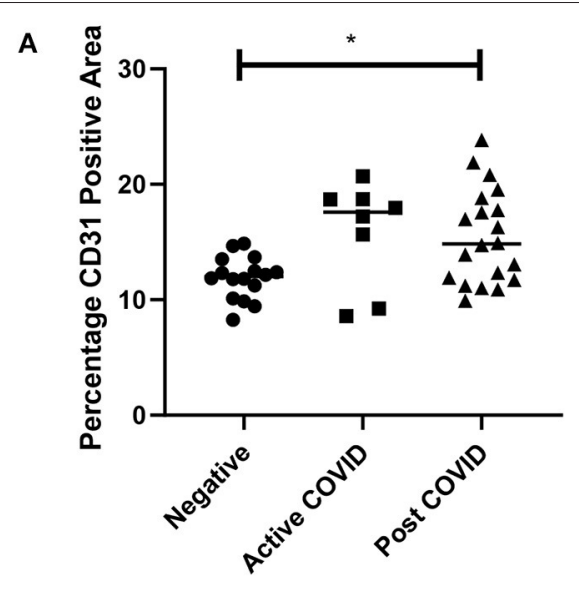

B

Negative

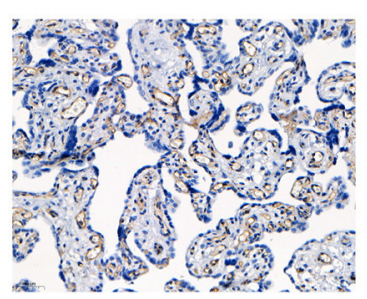

Active COVID

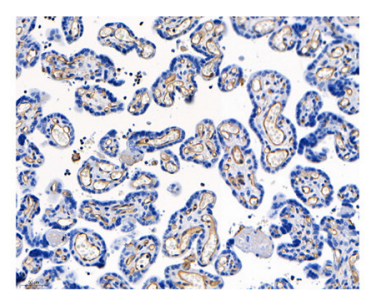

Post COVID

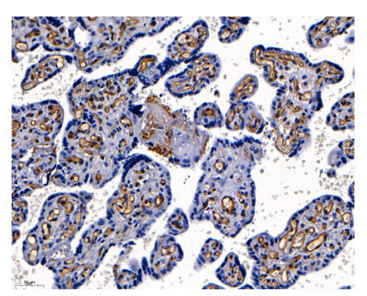

FIGURE 2 | Vascularity of placentas from women who had COVID-19 during their pregnancy. (A) There was a significant increase in the percentage vascularity in the placentas of women in the Post COVID group compared to controls $\left({ }^{*} p=0.026\right)$. There was no difference in placental vascularity in the Active COVID group compared to controls $(p=0.06$ ). (B) Representative image magnification X20. Kruskal-Wallis, Active COVID $n=8$, Post COVID $n=20$, and controls $n=16$.
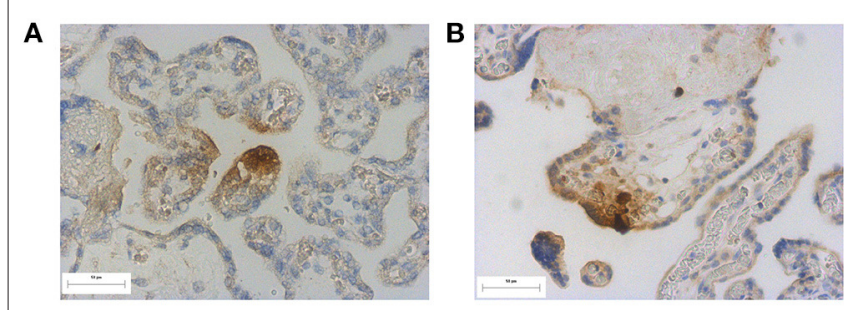

C
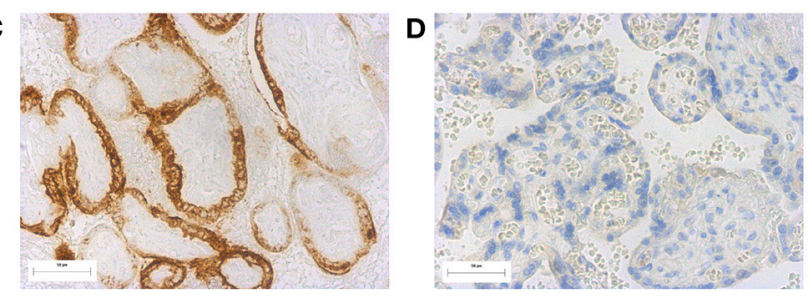

FIGURE 3 | SARS-CoV-2 spike staining in placentas (A) and (B) possible positive staining in a placenta from a participant within the active COVID group, (C) positive staining of a previously identified SARS-CoV-2 positive placenta (positive control) (D) negative SARS-CoV-2 spike protein staining in an unaffected placenta. Magnification X20. only four infants $\leq 10^{\text {th }}$ IBC in the Post COVID group. One of the roles of Hofbauer cells is to protect against pathogens, partly through expression of Toll-like Receptors (16). Hofbauer cell hyperplasia has been identified in 3 out of 20 SARS-CoV-2 positive placentas with an additional diagnosis of chronic histiocytic inter-villositis (CHI) (17). Hofbauer cell hyperplasia was not present in all $\mathrm{CHI}$ placentas, indicating that the increase was not due to CHI. The cause of the hyperplasia both in the study by Schwartz et al. and our study remain unknown. Further investigations into placental macrophage role/function following maternal SARS-CoV-2 infection are required to understand any potential adverse downstream placental changes as a result of the increase in Hofbauer cell number.

The number of macrophages may be linked to underlying pregnancy pathology as in the Active COVID group, one participant has diabetes mellitus and chronic hypertension, whilst another had gestational diabetes mellitus. In the Post COVID group, one participant had recurrent RFM, and one had hyperthyroidism. Previous research has identified an increase in the number of placental macrophages in the presence of maternal diabetes (type 1, 2 and gestational) (10). However, as the majority of participants in the Post COVID group did not have any 
A

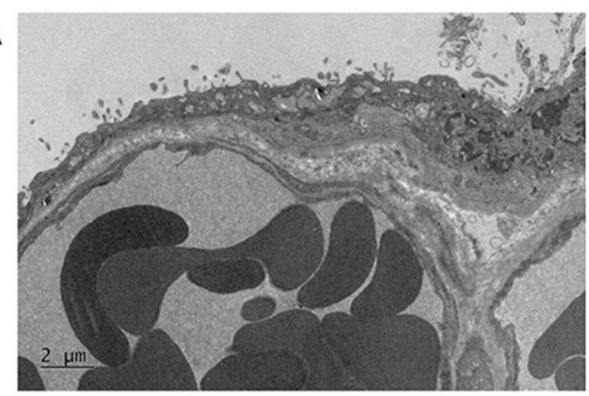

C

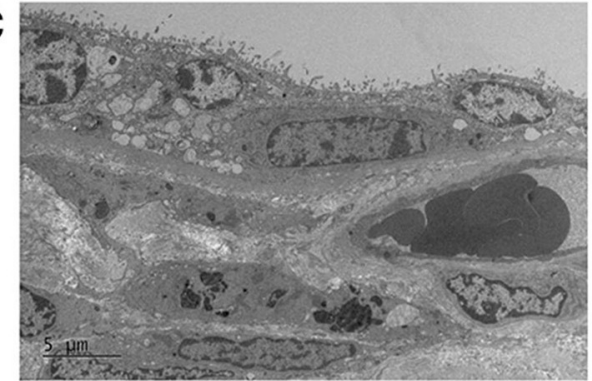

E

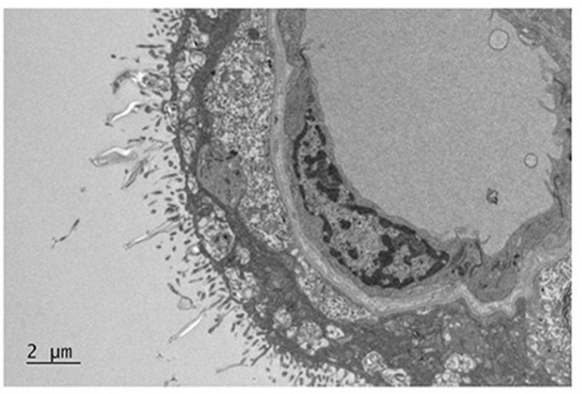

G

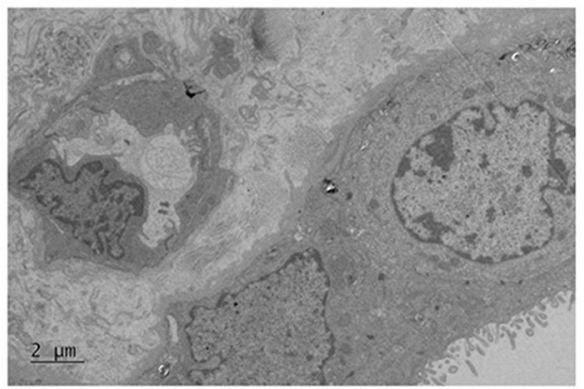

I

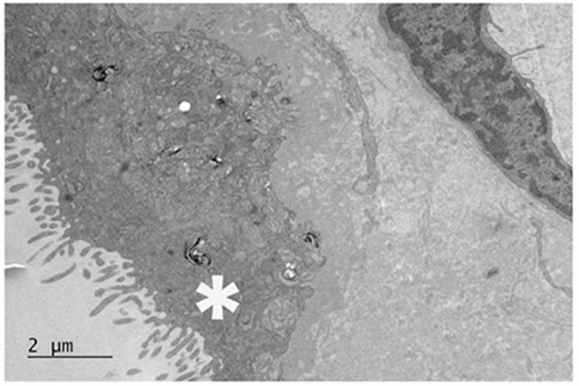

B

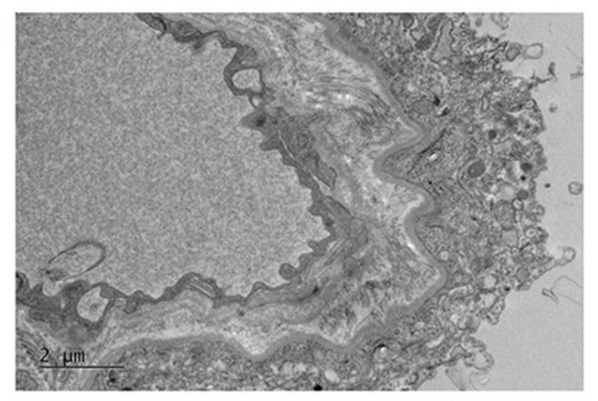

D

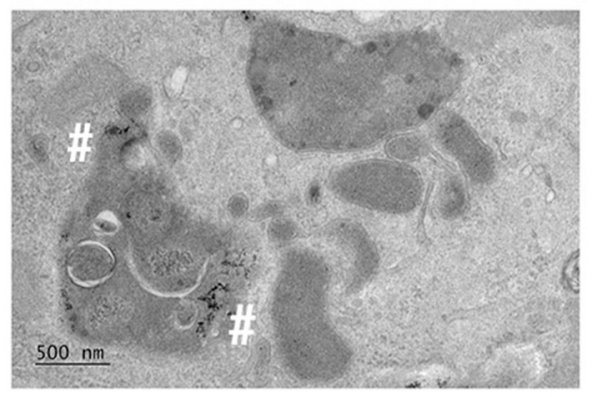

F

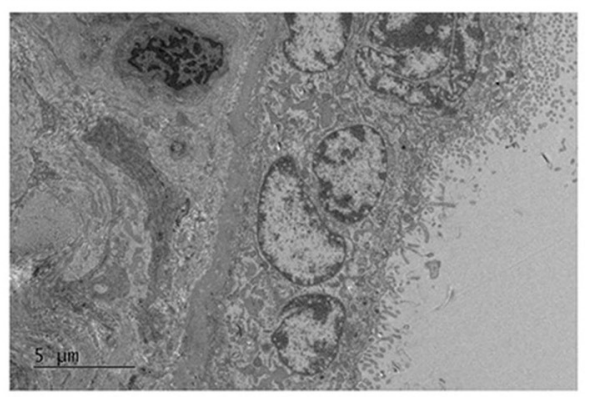

H

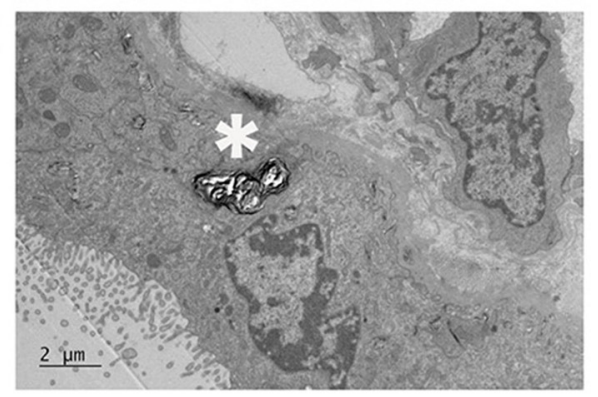

J

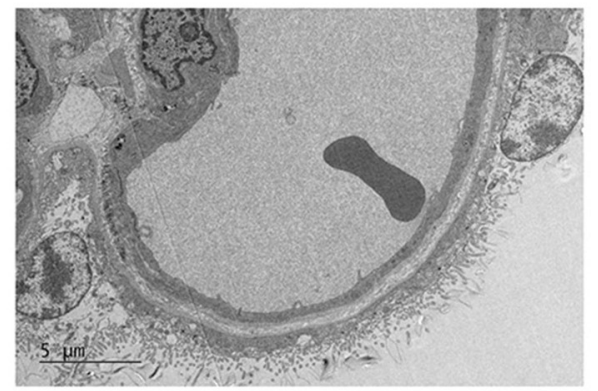

FIGURE 4 | Electron micrographs of placentas following maternal SARS-CoV-2 infection. Active COVID samples: (A) dilated cisternae of endoplasmic reticulum and sparce microvilli, (B) necrotic syncytium, (C) dilated syncytial endoplasmic reticulum, and swollen mitochondria, with multiple inclusions in an underlying Hofbauer cell and a multi-layered capillary basal lamina, (D) high power image of the ferritin particles within a Hofbauer cell inclusion. Post COVID samples: (E) large sub-apical vacuoles in the syncytiotrophoblast, (F) fibrotic stroma, thick basal lamina, malformed swollen villi, (G) small capillaries and (H) thickened basal lamina, swollen microvilli with possible loss of actin. Negative control samples: (I) numerous infoldings in the syncytial basal plasma membrane and thickened and cribriform basal lamina, with dispersed lamellar bodies (J) focal loss of microvilli (top right) and breakdown of the apical membrane and washed out syncytial cytoplasm (bottom left). The vasculosyncytial membrane appears relatively normal. *Indicates lamellar bodies, \# indicates ferritin particles. 
pregnancy complications, this is not likely to been the cause of the observed macrophage increase in all placentas.

\subsection{Poor Pregnancy Outcomes and Placental Pathology}

There are mixed findings as to whether maternal SARS-CoV-2 infection leads to an increased risk of poor pregnancy outcomes, particularly stillbirth. Whilst there are reports of an increased risk of stillbirth $(18,19)$, other studies have reported no difference $(20,21)$. There have been reports of an increase in neonatal deaths $(7,19)$; however, in our study, there were no poor pregnancy outcomes, although this is partly by design as women with Active COVID and a poor pregnancy outcome (e.g., a prior fetal death) were not approached to take part in the study.

Whilst we identified an increase in both maternal and fetal vascular malperfusion in our systematic review of studies reported between December 2019 and July 2020 (3), Girolamo et al., reported similar findings (22), and placental hypoperfusion and inflammation were identified in a more recent systematic review and meta-analysis. Other research has identified no associated placental pathology following third trimester infection, at either the macro- or microscopic level (23), or statistically significant increases in features, such as fibrin deposition (7). Our histopathology reports identified excessive syncytial knots, distal villous hypoplasia and villous immaturity in all study groups and there did not appear to be a preponderance of these features in women with SARSCoV-2 infection. Whilst there may be a placental pathology in pregnancies with a poor pregnancy outcomes, or declining fetal health in utero indicated by RFM (24-26), there does not appear to be any specific pathology in placentas from women with normal pregnancy outcomes, having mild symptoms and not requiring enhanced care in ICU. Although there was an increase in placental macrophages, this was not accompanied by an increase in other immune cells that are responsible for resolving viral infections, namely $\mathrm{T}$ and $\mathrm{B}$ lymphocytes (27). The syncytiotrophoblast acts as a placental barrier and prevents viruses passing from the maternal blood into the fetal circulation. Staining of placentas with the SARS-CoV-2 spike protein revealed that none of the samples evaluated here tested positive. As a result, an increase in immune cells would not be expected if there has not been a break in the syncytiotrophoblast.

Analysis of the placenta from the participants who tested positive for SARS-CoV-2 the day after delivery showed a low number of placental macrophages compared to the other placentas in the group (average of $2.97 \%$ across the three placental sections compared to between 8.67 and $22.98 \%$ for the remainder of the samples in the group). The most likely explanation for this difference is that the woman was possibly asymptomatic and was earlier in the course of COVID-19 infection, thereby reducing inflammation and consequent stress on the placenta.

There was a significant increase in vascularity between the Post COVID group compared to healthy controls. The high proportion of cases with fetal vascular malperfusion reported in the literature would have reduced the number of fetal vessels, whereas there was an increase in the Post COVID group and a trend toward an increase in the Active COVID group. It is likely that this observation in the Active COVID group may be affected by confounding factors, as the Active COVID group contained one infant who was large-for-gestational-age (LGA), two women with maternal diabetes (one with gestational diabetes) and one SGA infant. As the interval between a positive maternal COVID19 test and delivery was between 2 and 12 days, it would be unlikely that there would be sufficient time for additional capillaries to develop in the placenta, in response to maternal SARS-CoV-2 infection, despite angiogenesis continuing until term (28), however, an increase in vascularisation has previously been observed in pregnancies with diabetes mellitus (10). The increase in vascularity in the Post COVID group is less likely to be affected by confounding factors as only two women had additional pregnancy pathologies; one women had RFM during her pregnancy and another had hyperthyroidism. However, this may be a chance finding, as there was no statistically significant difference in the histopathological findings.

The placentas analyzed in our study were collected between May 2020 and May 2021. During this period, women in the UK were recommended to shield as they were classed as being clinically vulnerable and SARS-CoV-2 vaccinations for pregnant women were not approved by the Joint Committee on Vaccination and Immunization (JCVI) until April 2021 (29). Maternal vaccination has been found to reduce severity of SARSCoV-2 infection (30), and should be encouraged. Whilst our cohort of women did not have severe symptoms of COVID-19, despite being unvaccinated, the lack of any associated placental pathology and the minimal changes observed in the number of placental immune cells indicates that mild maternal COVID19 infection is unlikely to cause harm to the developing fetus. This study demonstrates that in general, women who have mild symptoms are unlikely to have a poor pregnancy outcome, although why some women do go onto have a poor outcome remains unclear.

None of the placentas sent for SARS-CoV-2 spike protein staining showed definitive positive immunostaining, indicating that in most cases SARS-CoV-2 does not enter the placenta itself, unlike other viral infections which manage to cross the syncytium including ZIKV (causing Zika virus disease). The mechanism of cellular entry for SARS-CoV-2 is thought to be mediated by TMPRSS2 and ACE2 receptor, with minimal co-expression found in the placenta and chorioamniotic membranes (31). Another study reported strong staining using immunohistochemistry for ACE2 in the placenta, particularly in the syncytiotrophoblast, but there was minimal staining of TMPRSS2 and any identifiable weak staining was on the endothelium of the villous capillaries (9). ZIKV does infect Hofbauer cells (32) and can replicate inside them (6). Hofbauer cells, however, have been shown not to express ACE2 (9) and, therefore, it would be unlikely for SARSCoV-2 to replicate similarly to ZIKV, even if there is a break in the syncytiotrophoblast to allow viral entry to the villous core.

There are multiple antibodies that can be used to identify positive SARS-CoV-2 staining in the placenta. We chose to use an antibody against the SARS-CoV-2 spike protein (clone 1A9) which has also been used by (33-35). However, alternative 
clones are available, such as 007 used by Facchetti et al. (36). Alternatively, antibodies for the SARS-CoV-2 nucleocapsid protein can also be used, such as those used by Debelenko et al. (35), Facchetti et al. (36), and Morotti et al. (37). Positive staining of the placenta has been identified using antibodies against the SARS-CoV-2 spike protein and the nucleocapsid.

\subsection{Strengths and Limitations}

The inclusion of women who had active COVID-19 infection at the time of delivery is a strength of this study, as it allows for any acute impact of maternal infection to be observed. We also collected contemporaneous healthy controls which is important as this provides controls for confounding issues such as the background stress of having a pregnancy during a pandemic (38), alongside other pregnancy complications such as diabetes mellitus. Ultrastructural analyses of a subset of placentas was also conducted. Whilst we were not able to identify any specific disease phenotype, one Post COVID placenta had a breakdown of the microvilli and another had several large sub-apical vacuoles in the syncytiotrophoblast which could be similar to the virion containing membrane-bound cisternal spaces described by Sisman et al. (39); however, no virion-like particles were seen within. None of the placentas had any obvious pathology.

We were able to include women in the Post COVID group of this study who had COVID-19 infection during their second or third trimester of pregnancy. Including these women along with women who tested positive in the third trimester, allowed for us to investigate whether maternal infection earlier in pregnancy resulted in morphological changes in the placentas, as this may be the point in gestation in which any insult to the placenta will have the largest effect. However, we did not see any specific pathology in the placentas of the women who had SARS-CoV-2 infection in the second or third trimester, possibly due to reduced severity of disease symptoms.

One of the limitations of this study is the uncertainty about whether the mothers in the control group had COVID-19 during their pregnancy, as antibody testing has not been implemented in the UK. In a recent survey of UK households, from December 2020 to July 2021, between 39 and $50 \%$ of people testing positive for COVID-19 were asymptomatic (40), therefore, there is the possibility that some of the women may have had asymptomatic COVID-19 during their pregnancy. Finally, no women included in the study has severe maternal disease, i.e., were not admitted to an intensive care unit due to COVID-19 infection, and there were no adverse outcomes such as stillbirth. Inclusions of cases with the most severe symptoms and signs may best allow for the correlation between maternal infection and placental dysfunction to be determined, as these infections may have had more of an impact on the placenta, for example as a result of severe maternal hypoxia.

\subsection{Conclusions}

In our cohort of women, with otherwise healthy pregnancy outcomes, there was no specific placental pathology associated with maternal SARS-CoV-2 infection during pregnancy. The observed increase in the number of placental macrophages warrants further investigation to see if these cells are responding to any possible maternal hypoxia affecting the placenta (which increases cytokine release from placental tissue). Analysis of cytokines in both maternal and cord blood, and placental samples would provide information as to the inflammatory status following maternal SARS-CoV-2 infection. Further analysis of placental samples, such as the examination of any differences in cell proliferation and apoptosis, would allow for any subtle changes to be found. Placentitis may be a phenotype only observed in pregnancies with a poor pregnancy outcome, but it does not appear to be present following mild maternal SARSCoV-2 infection.

\section{DATA AVAILABILITY STATEMENT}

The raw data supporting the conclusions of this article will be made available by the authors, without undue reservation.

\section{ETHICS STATEMENT}

Research ethical approval was given (18/NW/0451). The patients/participants provided their written informed consent to participate in this study.

\section{AUTHOR CONTRIBUTIONS}

MS conceived the study, performed immuno/histochemical staining, image analysis, statistical analysis, and manuscript preparation. AG helped perform immunohistochemical staining and image analysis. EA helped perform immunohistochemical staining. CJ prepared semithin sections of placentas submitted for TEM and provided analysis of EM images. GB performed histopathological analysis of placentas. $\mathrm{AH}$ conceived the study, provided guidance for laboratory work and manuscript preparation. All authors contributed to the article and approved the submitted version.

\section{FUNDING}

This work was supported by the Medical Research Council (Grant number MR/N010892/1, MS and AH), Tommy's Baby Charity (AH).

\section{ACKNOWLEDGMENTS}

We would like to thank the Department of Pathology, Alder Hey Children's Hospital for immunostaining placental samples for the spike protein of SARS-CoV-2 and the staff in the EM Core Facility in the Faculty of Biology, Medicine and Health for their assistance, and the Wellcome Trust for equipment grant support to the EM Core Facility.

\section{SUPPLEMENTARY MATERIAL}

The Supplementary Material for this article can be found online at: https://www.frontiersin.org/articles/10.3389/fviro. 2022.813312/full\#supplementary-material 


\section{REFERENCES}

1. World Health Organization. WHO Coronavirus (COVID-19) Dashboard. (2021). Available online at: https://covid19.who.int/

2. Chmielewska B, Barratt I, Townsend R, Kalafat E, Meulen Jvd, Gurol-Urganci I, et al. Effects of the COVID-19 pandemic on maternal and perinatal outcomes: a systematic review and meta-analysis. Lancet Glob Health. (2021) 9:e759-e772. doi: 10.1016/S2214-109X(21)00079-6

3. Sharps MC, Hayes DJL, Lee S, Zou Z, Brady CA, Almoghrabi Y, et al. A structured review of placental morphology and histopathological lesions associated with SARS-CoV-2 infection. Placenta. (2020) 11:13-29. doi: 10.1016/j.placenta.2020.08.018

4. Dunkelberger JR, Song WC. Complement in host immunity 34 Complement and its role in innate and adaptive immune responses. Cell Res. (2009) 20:34-5034. doi: 10.1038/cr.2009.139

5. Castellucci M, Celona ' A, Bartels "H, Steininger 'B, Benedettob V, Kaufmann 'P, et al. Mitosis of the hofbauer cell: possible implications for a fetal macrophage. Placenta. (1987) 8:65-76. doi: 10.1016/0143-4004(87)90040-3

6. Bhatnagar J, Rabeneck DB, Martines RB, Reagan-Steiner S, Ermias Y, Estetter LBC, et al. Zika virus RNA replication and persistence in brain and placental tissue. Emerg Infect Dis. (2017) 23:405-14. doi: 10.3201/eid2303.161499

7. Rebutini PZ, Zanchettin AC, Stonoga ETS, Prá DMM, de Oliveira ALP, Dezidério FdS, et al. Association between COVID-19 pregnant women symptoms severity and placental morphologic features. Front Immunol. (2021) 5:1893. doi: 10.3389/fimmu.2021.685919

8. Gao L, Ren J, Xu L, Ke X, Xiong L, Tian X, et al. Placental pathology of the third trimester pregnant women from COVID-19. Diagn Pathol. (2021) 16:1-11. doi: 10.1186/s13000-021-01067-6

9. Hecht JL, Quade B, Deshpande V, Mino-Kenudson M, Ting DT, Desai N, et al. SARS-CoV-2 can infect the placenta and is not associated with specific placental histopathology: a series of 19 placentas from COVID-19-positive mothers. Mod Pathol. (2020) 33:1. doi: 10.1038/s41379-020-0639-4

10. Kerby A, Shingleton D, Batra G, Sharps MC, Baker BC, Heazell AEP. Placental morphology and cellular characteristics in stillbirths in women with diabetes and unexplained stillbirths. Arch Pathol Lab Med. (2021) 145:82-9. doi: 10.5858/arpa.2019-0524-OA

11. Khong TY, Mooney EE, Ariel I, Balmus NCM, Boyd TK, Brundler MA, et al. Sampling and definitions of placental lesions Amsterdam placental workshop group consensus statement. In: Archives of Pathology and Laboratory Medicine. Vol. 140. (2016). p. 698-713.

12. Vracko R, Benditt EP. Capillary basal lamina thickening : its relationship to endothelial cell death and replacement. J Cell Biol. (1970) 47:281.

13. Benirschke K, Burton GJ, Baergen RN. Histopathological approach to villous alterations. In: Pathology of the Human Placenta. Berlin: Springer (2012). p. 395-410.

14. Derricott H, Jones RL, Greenwood SL, Batra G, Evans MJ, Heazell AEP. Characterizing villitis of unknown etiology and inflammation in stillbirth. Am J Pathol. (2016) 186:952-61. doi: 10.1016/j.ajpath.2015.12.010

15. Sharps MC, Baker BC, Guevara T, Bischof H, Jones RL, Greenwood SL, et al. Increased placental macrophages and a pro-inflammatory profile in placentas and maternal serum in infants with a decreased growth rate in the third trimester of pregnancy. Am J Reprod Immunol. (2020) 84:e13267. doi: 10.1111/aji.13267

16. Pudney J, He X, Masheeb Z, Kindelberger DW, Kuohung W, Ingalls RR. Differential expression of toll-like receptors in the human placenta across early gestation. Placenta. (2016) 46:1-10. doi: 10.1016/j.placenta.2016.07.005

17. Schwartz DA, Hyg M, Baldewijns M, Benachi A, Bugatti M, Bulfamante G, et al. Hofbauer cells and COVID-19 in pregnancy: molecular pathology analysis of villous macrophages, endothelial cells, and placental findings from 22 placentas infected by SARS-CoV-2 with and without fetal transmission. Arch Pathol Lab Med. (2021) 145:1328-40. doi: 10.5858/arpa.20210296-SA

18. Khalil A, Dadelszen PV, Draycott T, Ugwumadu A, O’Brien P, Magee L. Change in the incidence of stillbirth and preterm delivery during the COVID19 pandemic. JAMA. (2020) 324:705-6. doi: 10.1001/jama.2020.12746

19. Gurol-Urganci I, Jardine JE, Carroll F, Draycott T, Dunn G, Fremeaux A, et al. Maternal and perinatal outcomes of pregnant women with SARS-CoV-2 infection at the time of birth in England: national cohort study. Am J Obstet Gynecol. (2021) 225:522.e1-522.e11. doi: 10.1016/j.ajog.2021.05.016

20. Stowe J, Smith H, Thurland K, Ramsay ME, Andrews N, Ladhani SN. Stillbirths during the COVID-19 pandemic in England, April-June 2020. JAMA. (2021) 325:86-7. doi: 10.1001/jama.2020.21369

21. Bunnell ME, Koenigs KJ, Roberts DJ, Quade BJ, Hornick JL, Goldfarb IT Third trimester stillbirth during the first wave of the SARS-CoV-2 pandemic: similar rates with increase in placental vasculopathic pathology. Placenta. (2021) 109:72-4. doi: 10.1016/j.placenta.2021.04.003

22. Girolamo RD, Khalil A, Alameddine S, D'Angelo E, Galliani C, Matarrelli B, et al. Placental histopathology after SARS-CoV-2 infection in pregnancy: a systematic review and meta-analysis. Am J Obstet Gynecol MFM. (2021) 3:100468. doi: 10.1016/j.ajogmf.2021.100468

23. Tasca C, Rossi RS, Corti S, Anelli GM, Savasi V, Brunetti F, et al. Placental pathology in COVID-19 affected pregnant women: a prospective case-control study. Placenta. (2021) 110:9-15. doi: 10.1016/j.placenta.2021.04.002

24. Linehan L, O’Donoghue K, Dineen S, White J, Higgins JR, Fitzgerald B. SARS-CoV-2 placentitis: an uncommon complication of maternal COVID-19. Placenta. (2021) 104:261-6. doi: 10.1016/j.placenta.2021.01.012

25. Watkins JC, Torous VF, Roberts DJ. Defining severe acute respiratory syndrome coronavirus 2 (SARS-CoV-2) placentitis: a report of 7 cases with confirmatory in situ hybridization, distinct histomorphologic features, and evidence of complement deposition. Arch Pathol Lab Med. (2021) 145:1341-9. doi: 10.5858/arpa.2021-0246-SA

26. Marton T, Hargitai B, Hunter K, Pugh M, Murray P. Massive Perivillous Fibrin Deposition and Chronic Histiocytic Intervillositis a Complication of SARS-CoV-2 Infection:. Pediatr Develop Pathol. (2021) 24:450-4. doi: 10.1177/10935266211020723

27. Cox RJ, Brokstad KA. Not just antibodies: $B$ cells and $T$ cells mediate immunity to COVID-19. Nat Rev Immunol. (2020) 20:581-2. doi: 10.1038/s41577-020-00436-4

28. Charnock-Jones DS, Kaufmann P, Mayhew TM. Aspects of human fetoplacental vasculogenesis and angiogenesis. I. molecular regulation. Placenta. (2004) 25:103-13. doi: 10.1016/j.placenta.2003.10.004

29. Public Health England. JCVI Issues New Advice on COVID-19 Vaccination for Pregnant Women - GOV.UK. (2021). Available online at: https://www.gov. uk/government/news/jcvi-issues-new-advice-on-covid-19-vaccination-forpregnant-women

30. Morgan JA, Biggio JRJ, Martin JK, Mussarat N, Chawla HK, Puri $\mathrm{P}$, et al. Maternal outcomes after severe acute respiratory syndrome Coronavirus 2 (SARS-CoV-2) infection in vaccinated compared with unvaccinated pregnant patients. Obstet Gynecol. (2021). 139:107-9. doi: 10.1097/AOG.0000000000004621

31. Pique-Regi R, Romero R, Tarca AL, Luca F, Xu Y, Alazizi A, et al. Does the human placenta express the canonical cell entry mediators for sars-cov-2? eLife. (2020) 9:1-15. doi: 10.7554/eLife.58716

32. Tabata T, Petitt M, Puerta-Guardo H, Michlmayr D, Wang C, Fang-Hoover J, et al. Zika virus targets different primary human placental cells, suggesting two routes for vertical transmission. Cell Host Microbe. (2016) 20:155-66. doi: 10.1016/j.chom.2016.07.002

33. Smithgall MC, Liu-Jarin X, Hamele-Bena D, Cimic A, Mourad M, Debelenko L, et al. Third trimester placentas of (SARS-CoV-2)-positive women: histomorphology, including viral immunohistochemistry and in situ hybridization. Histopathology. (2020) 77:994-9. doi: 10.1111/his.14215

34. Pulinx B, Kieffer D, Michiels I, Petermans S, Strybol D, Delvaux S, et al. Vertical transmission of SARS-CoV-2 infection and preterm birth. Eur J Clin Microbiol Infect Dis. (2020) 39:2441-5. doi: 10.1007/s10096-02003964-y

35. Debelenko L, Katsyv I, Chong AM, Peruyero L, Szabolcs M, Uhlemann AC. Trophoblast damage with acute and chronic intervillositis: disruption of the placental barrier by severe acute respiratory syndrome coronavirus 2 . Hum Pathol. (2021) 109:69-79. doi: 10.1016/j.humpath.2020.12.004

36. Facchetti F, Bugatti M, Drera E, Tripodo C, Sartori E, Cancila V, et al. SARS-CoV2 vertical transmission with adverse effects on the newborn revealed through integrated immunohistochemical, electron microscopy and molecular analyses of Placenta. EBioMedicine. (2020) 59:102951. doi: 10.1016/j.ebiom.2020.102951 
37. Morotti D, Cadamuro M, Rigoli E, Sonzogni A, Gianatti A, Parolin C, et al. Molecular pathology analysis of SARS-CoV-2 in syncytiotrophoblast and hofbauer cells in placenta from a pregnant woman and fetus with COVID-19. Pathogens. (2021) 10:479. doi: 10.3390/pathogens 100 40479

38. Brien ME, Bouron-Dal Soglio D, Dal Soglio S, Couture C, Boucoiran I, Nasr Y, et al. Pandemic stress and SARS-CoV-2 infection are associated with pathological changes at the maternal-fetal interface. Placenta. (2021) 115: 37-44. doi: 10.1016/j.placenta.2021.09.007

39. Sisman J, Jaleel MA, Moreno W, Rajaram V, Collins RRJ, Savani RC, et al. Intrauterine transmission of SARS-CoV-2 infection in a preterm infant. Pediatr Infect Dis J. (2020) 39:e265-e267. doi: 10.1097/INF.00000000000 02815

40. Office for National Statistics. Coronavirus (COVID-19) Infection Survey, Characteristics of People Testing Positive for COVID-19, UK. (2021). Available online at: https://www.ons. gov.uk/peoplepopulationandcommunity/healthandsocialcare/conditionsand diseases/datasets/coronaviruscovid19infectionsinthecommunityinengland
Conflict of Interest: The authors declare that the research was conducted in the absence of any commercial or financial relationships that could be construed as a potential conflict of interest.

Publisher's Note: All claims expressed in this article are solely those of the authors and do not necessarily represent those of their affiliated organizations, or those of the publisher, the editors and the reviewers. Any product that may be evaluated in this article, or claim that may be made by its manufacturer, is not guaranteed or endorsed by the publisher.

Copyright (๑) 2022 Sharps, Garrod, Aneni, Jones, Batra and Heazell. This is an open-access article distributed under the terms of the Creative Commons Attribution License (CC BY). The use, distribution or reproduction in other forums is permitted, provided the original author(s) and the copyright owner(s) are credited and that the original publication in this journal is cited, in accordance with accepted academic practice. No use, distribution or reproduction is permitted which does not comply with these terms. 